\title{
¿Beneficia el deporte de alto rendimiento a las personas con esclerosis múltiple? Estudio de caso en ciclismo adaptado
}

\section{Does high performance sport benefit people with multiple sclerosis? Case study in para-cycling}

Fecha de recepción: 18-06-2019

Fecha de aceptación: 10-12-2019
Antonio Moreno-Tenas

Grupo de Trabajo de Psicología del Deporte del Col.legi Oficial de Psicologia de la Comunitat Valencia

\section{resumen/alsstract:}

La esclerosis múltiple (EM), por sus síntomas, es un problema de salud que la Unión Ciclista Internacional reconoce como una de las causas de discapacidad reconocidas para poder competir en ciclismo adaptado. La EM se caracteriza por producir trastornos sensitivos-motores, neuritis óptica, diplopía, ataxia, vejiga neurogénica, fatiga, disartria, síntomas paroxísticos, afasia, apraxia, convulsiones, demencias y signos extrapiramidales como corea y rigidez. La actividad física reduce el número de recaídas clínicas y se correlaciona con menores limitaciones funcionales y menos síntomas de la enfermedad. En el presente estudio de caso en el que se interviene con un handbiker, se observa que el ejercicio físico de alto rendimiento podría tener algún tipo de relación con mejorías en los síntomas de la enfermedad y en la capacidad del organismo para asimilar el, y adaptarse al, esfuerzo.

Multiple sclerosis (MS), due to its symptoms, is a health problem that the International Cycling Union recognizes as one of the recognized causes of disability to compete in paracycling. MS is characterized by producing sensory-motor disorders, optic neuritis, diplopia, ataxia, neurogenic bladder, fatigue, dysarthria, paroxysmal symptoms, aphasia, apraxia, seizures, dementias and extrapyramidal signs such as chorea and stiffness. Physical activity reduces the number of clinical relapses and correlates with lower functional limitations and fewer symptoms of the disease. In the present case study in which a handbiker is intervened, it is observed that high performance physical exercise could have some kind of relationship with improvements in the symptoms of the disease and in the body's ability to assimilate and adapt to the effort.

\section{palahras clave/keywords:}

Ciclismo adaptado, esclerosis múltiple, alto rendimiento, ejercicio físico, dolor crónico

Paracycling, multiple sclerosis, high performance, physical exercise, chronic pain

\section{INTRODUCCIÓN}

La perspectiva de salud en Psicología del Deporte está presente en todos sus ámbitos de actuación, entre ellos en el alto rendimiento y en poblaciones especiales (Cantón, 2016). Ambos convergen en la práctica competitiva de ciclismo adaptado, regulada por la Unión Ciclista Internacional (en adelante UCI), a través del Reglamento del Deporte Ciclista (UCI, 2019). En su título XVI dedicado al paraciclismo, establece que este tipo de pruebas se dirigen exclusivamente a deportistas que tengan una discapacidad calificada que sea permanente y que cumpla con unos criterios mínimos de deficiencia, establecidos por la propia UCI. 
Las corredoras y corredores son evaluados en base a ocho tipos de deficiencias elegibles: disminución de la fuerza muscular, deterioro de movimientos, deficiencia de los miembros, diferencia de longitud de las piernas, hipertonía, ataxia, atetosis y deficiencia visual. Al menos uno de los tipos elegibles de discapacidad enumerados debe estar presente y ser consecuencia directa de un problema de salud permanente.

Las pruebas de ciclismo adaptado se organizan en diferentes divisiones o modalidades deportivas: 1) bicicleta a mano $(\mathrm{H}), 2)$ triciclo $(\mathrm{T}), 3$ ) bicicleta $(\mathrm{C})$ y 4$)$ tándem $(\mathrm{B})$; y éstas, a su vez, se clasifican en clases deportivas en las que los corredores y corredoras son agrupados en la medida en que la discapacidad les afecta en tareas y en actividades fundamentales para el paraciclismo. En el caso de la División Handbike (bicicletas a mano), los ciclistas que compiten utilizando bicicletas de mano propulsadas por los brazos o bien de tronco y brazo, son agrupados en las clases H1-H4, siendo en ambos casos obligatoria la posición supina; aquellos que lo hacen en posición de rodillas, conforman la clase H5.

Uno de los problemas de salud subyacentes que pueden resultar en una discapacidad elegible es la esclerosis múltiple (en adelante EM). Se trata de una enfermedad desmielinizante, crónica, autoinmune e inflamatoria que afecta a todo el sistema nervioso central (Ebers, 2008). La EM se caracteriza por trastornos sensitivos-motores en uno o más miembros, neuritis óptica, diplopía, ataxia, vejiga neurogénica, fatiga, disartria y síntomas paroxísti$\cos$. En menor medida afasia, apraxia, convulsiones, demencias y signos extrapiramidales como corea y rigidez (Ruíz y Solar, 2006).

Existen numerosas evidencias de los beneficios que el ejercicio físico conlleva para la salud física y psicológica de las personas con EM (Negaresh, Motl, Zimmer, Mokhtarzade y Baker, 2019; Dalgas, Stenager y Ingemann-Hansen, 2008). La mayoría de las guías informativas editadas en España dirigidas a personas con EM y a sus familiares recomiendan la práctica de ejercicio físico como parte importante del tratamiento, tanto para prevenir y mejorar los síntomas y las funciones que puedan verse deterioradas por la inactividad, como para contribuir al bienestar general (Esclerosis Múltiple España; FEDEMA, 2015).

¿Podría el ejercicio físico de alto rendimiento empeorar los síntomas o la progresión de la EM? Los estudios no encuentran relación entre la actividad física y las recaídas clínicas (Tallner et. al., 2011), incluso parecen sugerir lo contrario, que la reducción de la actividad física podría ser un correlato conductual, aunque no necesariamente causa, de la progresión de la discapacidad a corto plazo en personas con esclerosis múltiple (Molt y McAuley, 2011).

Y, en el caso de que no los empeorara, ¿podría entonces el ejercicio físico de alto rendimiento mejorar los síntomas de las personas con EM? La literatura científica informa que aquellos pacientes que aumentan sus niveles de ejercicio físico experimentan un menor cambio en las limitaciones funcionales (Stuifbergen, Blozis, Harrison y Becker, 2006). Otros estudios sugieren que cuanto más elevado es el nivel de actividad física las personas con EM refieren menos síntomas de la enfermedad (Motl, Snook, McAuley y Gliottoni, 2006). Incluso en entrenamientos con sobrecarga, las personas con EM, independientemente de los diferentes niveles de diversidad funcional, mostraron una mejora en fuerza muscular (Filipi, Kucera. Filipi, Ridpath y Leuschen, 2011). 
Una búsqueda sistemática sobre la relación entre el ejercicio físico y la progresión de la enfermedad no ha podido establecer si el ejercicio tiene un efecto modificador de la misma (Dalgas y Stenager, 2012).

El presente estudio de caso tiene como objetivo mostrar los cambios producidos en algunos de los parámetros evaluados en un ciclista adaptado de alto rendimiento y diagnosticado de Esclerosis Múltiple tras la intervención psicológica realizada para optimizar su rendimiento. DESCRIPCIÓN DEL CASO

El sujeto sobre el que se realiza el presente estudio es un hombre, de 45 años, diagnosticado de esclerosis múltiple a los 41 años. Refiere problemas motores, de equilibrio y de coordinación, espasticidad, fatiga, alteraciones de la sensibilidad y dolores, entre otros.

En 2014 comienza a tener problemas para caminar y precisa de la ayuda de un bastón. Su práctica deportiva en bicicleta todo terreno pronto requiere de la incorporación de un motor para que el pedaleo asistido alivie el dolor que empieza a experimentar en una pierna. Progresivamente va perdiendo fuerza en los dos miembros inferiores, hasta que, en 2015, debe utilizar permanentemente una silla de ruedas para desplazarse.

Ese mismo año empieza a participar en competiciones de ciclismo adaptado con una bicicleta a mano (o handbike), pese a verbalizaciones del tipo "el neurólogo me dijo que, tal y como avanzaba la enfermedad, no iba a poder competir."

Sin embargo, en las temporadas siguientes mejora sus resultados en las principales competiciones autonómicas y nacionales de su categoría, llegando a alcanzar un nivel competitivo tal que suele disputar de forma habitual los primeros puestos en aquellas carreras en las que participa y lograr la victoria en algún campeonato.

Incluido en la "Master List" de la RFEC (relación oficial de ciclistas adaptados que han pasado un proceso clasificador), en noviembre de 2017 solicita la intervención psicológica por parte de un psicólogo del deporte con el objetivo de "mantener o mejorar" los resultados logrados en esa temporada.

\section{Evaluación}

La evaluación inicial, que se inicia tras haber sido informado y una vez que ha cumplimentado el consentimiento informado, se prolonga por dos sesiones de 90 y 70 minutos de duración respectivamente, separadas entre sí por dos semanas.

La evaluación se centra en conocer al sujeto, en precisar cuál es su demanda de actuación profesional, así como en explorar el impacto de la EM en su proyecto de vida y en relación a la práctica deportiva de alto rendimiento que realiza.

También se reflexiona sobre su objetivos deportivos, sus motivos para implicarse en una actividad física intensiva y para participar en competiciones, así como se miden y cuantifican variables psicológicas como la motivación o su estado de ánimo (Moreno-Tenas, 2019).

A continuación se le propone cumplimentar un registro de entrenamiento diario que incluye parámetros relacionados con la carga de trabajo (vatios promedio y training stress score, en adelante TSS), así como dos escalas ad hoc: una de Percepción Subjetiva de Esfuer- 
zo ( $0=$ Muy, muy suave; $1=$ Muy suave; $2=$ Suave; $3=$ Moderado; $4=$ Algo duro; 5,6=Duro; 7,8,9=Muy duro; $10=$ Muy, muy duro) y otra de Percepción Subjetiva de Dolor Pre-Post Ejercicio ( $0=$ Ausencia; $1,2,3=$ Leve ; 4,5,6=Moderado; 7,8,9=Intenso; $10=$ Insoportable).

La evaluación del estado afectivo se realiza mediante el "Perfil de Estados de Ánimo-breve" (Andrade, Arce, De Francisco, Torrado y Garrido, 2013), versión española de "Profile of Mood States" de McNair, Lorr y Droppleman (1971, 1992), con unos resultados de bondad de ajuste del modelo: $\chi 2(390)=803.12, \mathrm{p}<.01$; de .97 para CFI y para NNFI; de .051 para el índice RMSEA (IC 90\% [.046; .057]); y de .057 para SRMR.

La motivación se evalúa mediante la "Escala de Motivación Deportiva" (Balaguer, Castillo y Duda, 2007), versión española de "Sports Motivation Scale" de Pelletier, Fortier, Vallerand, Tuson, Brière y Blais (1995), con una fiabilidad del instrumento ya confirmada en anteriores estudios (Balaguer, Castillo y Duda, 2003; Núñez, Martín-Albo y Navarro, 2007).

A lo largo de la intervención se incorporan otros instrumentos, algunos de carácter general para evaluar las características psicológicas más relacionadas con el rendimiento deportivo, y otros para medir aspectos situacionales en entrenamientos y competiciones, como la ansiedad precompetitiva, las estrategias de afrontamiento utilizadas y sus experiencias óptimas (o estados de flow).

Para evaluar el estado de flow se utiliza la "Escala de Estado de Fluidez" (García, Jiménez, Santos-Rosa, Reina y Cervelló, 2008) versión española de "Flow State Scale” de Jackson y Marsh (1996), con valores en el alfa de Cronbach superiores a .70.

Todos ellos se utilizarán a lo largo de la intervención en función de las necesidades de ésta, para evaluar el grado de adquisición de las habilidades y destrezas psicológicas, así como para conocer los estados previos y posteriores a entrenamientos y competiciones.

\section{Intervención}

La intervención se realiza, desde el principio, a distancia. La comunicación se mantiene telefónicamente y, en ocasiones, a través de Skype. Para la visualización de los materiales de trabajo, la cumplimentación de registros y la administración de pruebas (documentos, hojas de cálculo y presentaciones) se utiliza la plataforma y los documentos de Google Drive.

Las sesiones, con una periodicidad quincenal, tienen una duración aproximada de $75 \mathrm{mi}$ nutos. Durante el transcurso de las mismas, se realizan anotaciones de la información más relevante que aporta. Las sesiones no son grabadas ni registradas de otro modo.

La intervención se estructura en cuatro amplios periodos: pretemporada 2017-18, periodo competitivo 2018, pretemporada 2018-19 y periodo competitivo 2019.

1.Periodo Precompetitivo I (Pretemporada de 2017-2018). Una vez realizada una primera evaluación para conocer los efectos de la enfermedad sobre su calidad de vida y su bienestar y tras explorar sus antecedentes de práctica deportiva y sus objetivos competitivos, se inicia la intervención psicológica, que se prolonga de diciembre de 2017 hasta febrero de 2018, con una periodicidad aproximada de dos/tres semanas entre sesiones. 
El sujeto estructura gran parte de su vida en base a la competición deportiva, lo que le lleva a realizar ejercicio físico de alto rendimiento, entrenando la mayor parte de los días de la semana y con un nivel de carga física elevado.

Cuenta con patrocinadores particulares que colaboran con él en la búsqueda y adquisición de los materiales necesarios para practicar este deporte, así como para que participe en competiciones. A éstas se desplaza en su propio vehículo adaptado.

En base a ello, se realiza un análisis de necesidades deportivas y se establecen los objetivos de intervención: análisis, discusión y desarrollo de metas realistas y centradas en la tarea, incremento de la motivación intrínseca, análisis del rendimiento y de aquellos aspectos que dependen de su ejecución, incremento de la autoconfianza.

2.Periodo competitivo I (Temporada 2018). Abarca el periodo que transcurre desde marzo a octubre, en el que se disputan las competiciones. Durante este periodo se mantiene la periodicidad de las sesiones en dos/tres semanas, con una duración aproximada de 70 minutos cada una.

A falta de una quincena para el inicio de las primeras carreras, se suceden varias semanas de entrenamientos con fuertes dolores neuropáticos, seguidos de una fractura en el pie y una lesión que le obliga a parar de entrenar para recuperarse, por lo que no disputa las carreras de la primera parte de la temporada.

La segunda parte se ve marcada por el fuerte viento que afectó durante meses a su zona geográfica, lo que dificulta los entrenamientos. En este periodo se suceden los periodos de baja motivación, frustración y también se mantiene la espasticidad muscular.

La intervención se centra en abordar el aumento de pensamientos negativos y creencias irracionales que generan las dificultades derivadas de los dolores y las condiciones meteorológicas; se interviene sobre el estado de ánimo, que sufre importantes variaciones; los objetivos se centran en los resultados que no llegan y en mantener un plan establecido a expensas de los acontecimientos que se producen y que impiden la consecución del mismo.

3.Periodo Precompetitivo II (Pretemporada 2018-2019). De nuevo, desde octubre se dirige la intervención durante una nueva pretemporada al establecimiento de objetivos de tarea, frente a la excesiva importancia de los resultados y la presencia en los primeros puestos. Asimismo, se incorpora a la planificación de los entrenamientos aspectos antecedentes a los estados de flow, con la finalidad de que su atención se focalice en las propias acciones y en su ejecución frente a los aspectos contextuales de la competición.

La potencia media que es capaz de desarrollar en los entrenamientos experimenta un incremento significativo (de los 154 y 168 vatios promedio de los entrenamientos de los meses de junio y julio de 2018 pasamos a 224 en enero y 246 en marzo de 2019).

4.Periodo Competitivo II (Temporada 2019). En el momento de redactar el presente artículo la temporada se encuentra en su meridiano, el rendimiento es óptimo y los entrenamientos y competiciones se estructuran en base al factor de intensidad y la concentración dirigida a sus propios parámetros. 
Únicamente circunstancias personales y relacionadas con la calidad de los materiales con los que entrena/compite provocan cambios significativos en su estado de ánimo, y que influyen en su motivación. Hasta la fecha se mantienen los objetivos centrados en la tarea, el mantenimiento y consolidación de creencias racionales, la optimización de su estado afectivo y la planificación de los entrenamientos y competiciones con objetivos de rendimiento establecidos junto a su preparador físico, que impliquen un desafío realista y cuya monitorización sea posible mediante aquellos parámetros propios que puedan proporcionar una retroalimentación constante e inmediata.

\section{Seguimiento}

La evolución de las actuaciones propuestas durante la intervención y valoradas a través de los instrumentos de evaluación descritos se realiza durante toda la intervención.

Todos los aspectos físicos y psicológicos relacionados con la intervención se evalúan con una frecuencia que varía desde aquellos cuya fluctuación es prácticamente diaria, como los relativos a los entrenamientos, pasando por aquellos que se concentran en periodos competitivos, hasta aquellos otros más estables que se evalúan con una periodicidad superior al mes o incluso anualmente, como es el caso de la motivación.

El seguimiento e intervención de las habilidades y destrezas psicológicas se prolonga por más de año y medio, y continúa actualmente.

Cabe destacar que, de las 47 asistencias hospitalarias de urgencia del año 2017, durante 2018 el seguimiento realizado ha contabilizado una considerable reducción, con 2 traslados a urgencias. Durante 2019, únicamente ha sido necesaria una sola asistencia sanitaria de urgencia.

\section{RESULTADOS}

El estado de ánimo experimenta constantes fluctuaciones durante la intervención. En un principio, el perfil del sujeto destaca por altas puntuaciones en aquellos aspectos que se relacionan negativamente con el rendimiento. En cambio, dos meses después, apenas unas semanas antes del inicio de las primeras competiciones, se aprecia el 'Perfil de Iceberg' (Cólera ha descendido de 8 a 0; Fatiga de 14 a 2; Tensión de 12 a 0; y Estado Deprimido de 16 a 1; mientras que los aspectos relacionados positivamente con el rendimiento han aumentado: Vigor pasa de 10 a 14 y Amistad de 11 a 20).

Las circunstancias personales y los acontecimientos y resultados deportivos afectan recurrentemente a su estado de ánimo. En esta ocasión, encontramos tres perfiles diferentes correspondientes a distintos momentos de 2018 y 2019. El primero de ellos es un perfil típico de pretemporada obtenido después de un entrenamiento con gran carga de trabajo, en el que la puntuación en Fatiga asciende hasta 14; el segundo de ellos, separado en apenas dos semanas del anterior, refleja una circunstancia personal adversa (Amistad desciende hasta un valor de 4 y Vigor de 8, mientras que el resto de puntuaciones negativas superan valores de 12, llegando Cólera y Estado Deprimido los 16). El tercer perfil refleja un conflicto interpersonal producido cuatro meses después del último registro. Una semana después se produciría la única recaída con ingreso hospitalario de este año. 
Figura 1. POMS-breve, Perfil de Estados de Ánimo

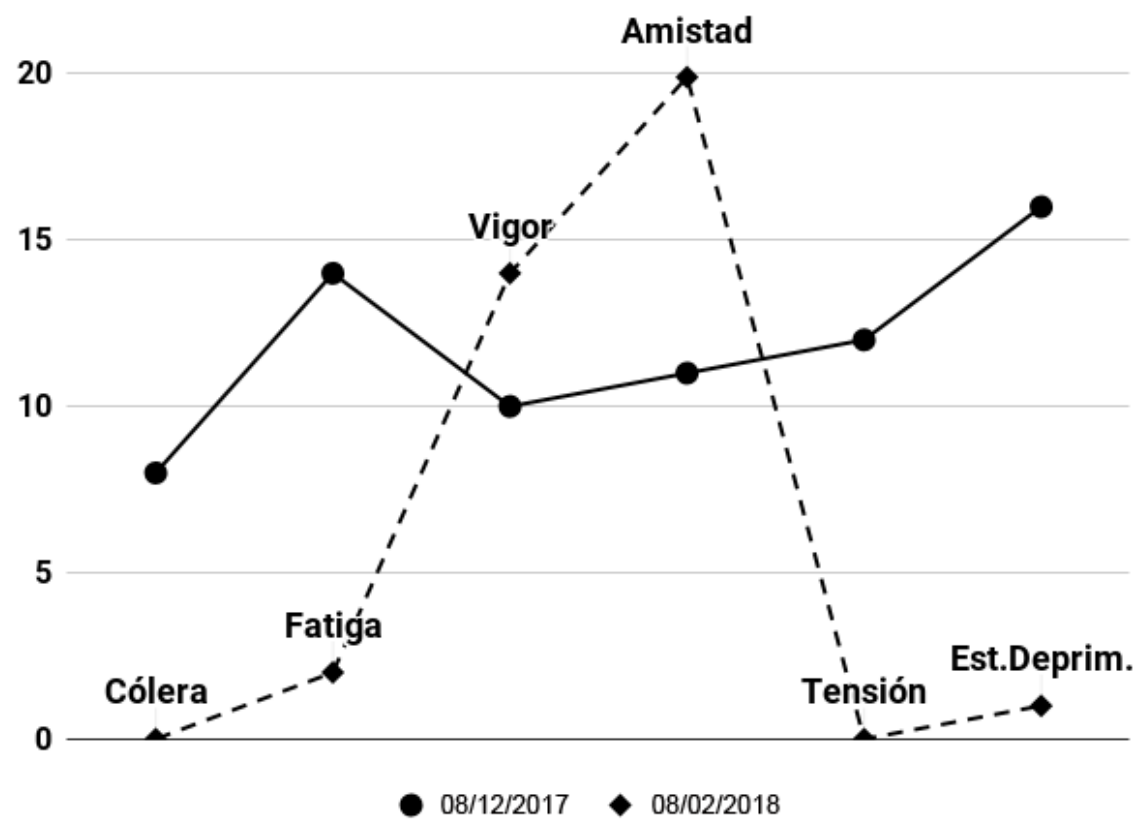

Figura 2. POMS-breve, Perfil de Estados de Ánimo

20

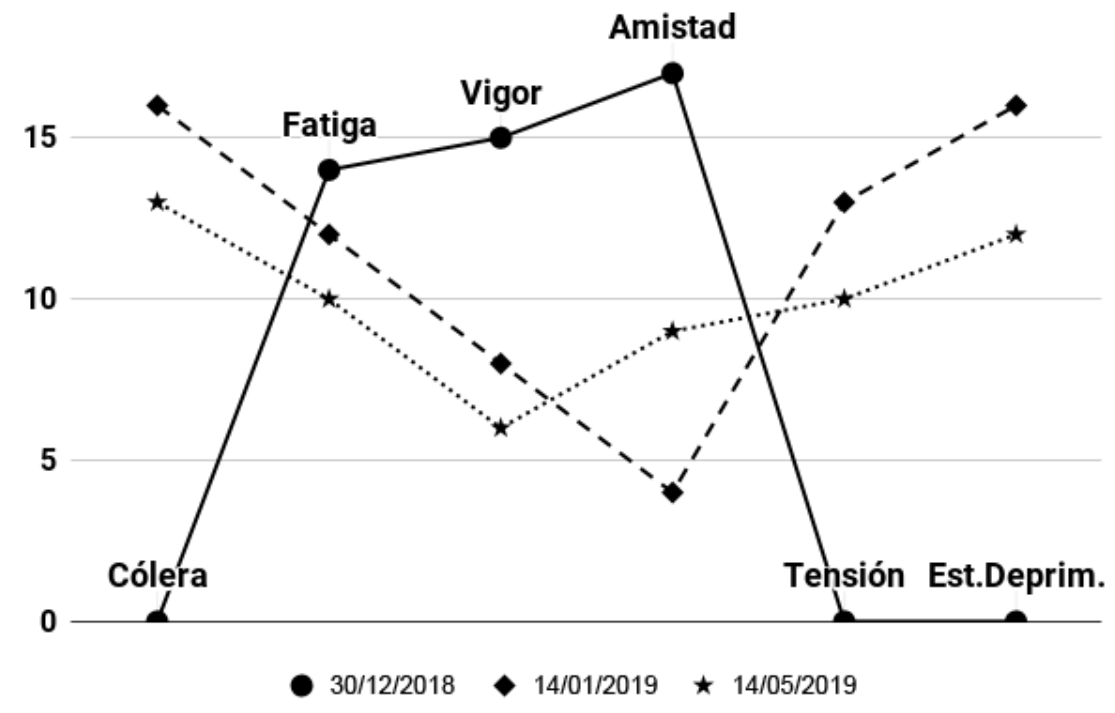




\section{praxis}

Figura 3. FSS, Flow State Scale

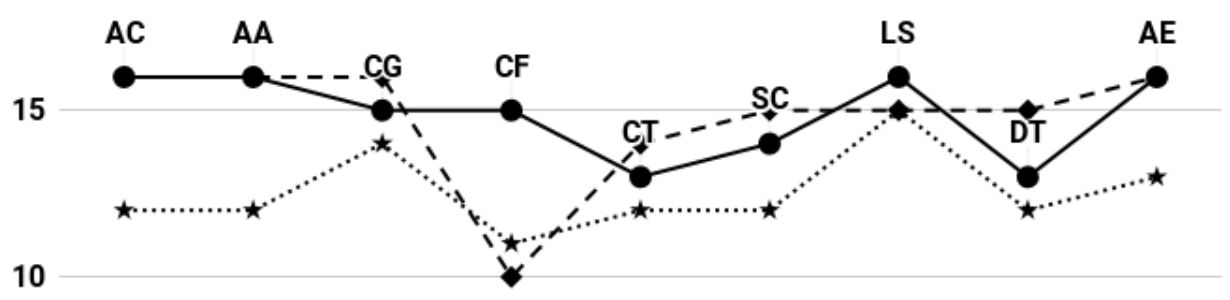

5

0

$24 / 10 / 2018 \diamond 28 / 10 / 2018 \star 05 / 03 / 2019$

Figura 4. Percepción de dolor antes y después del entrenamiento

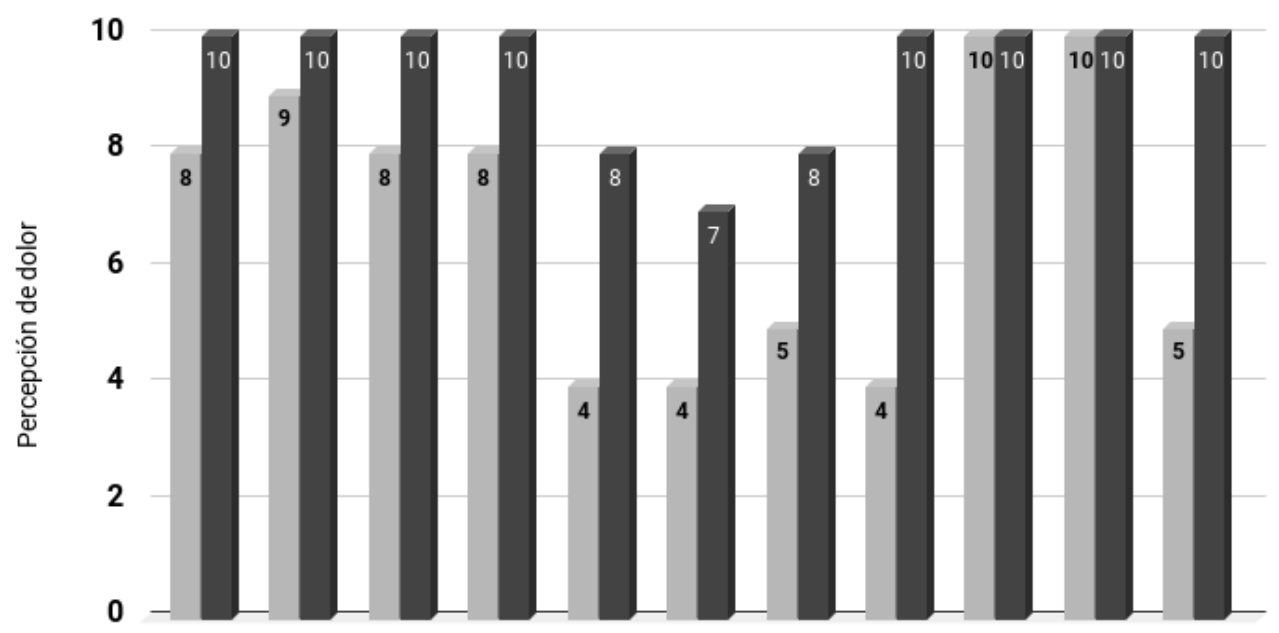

Febrero de 2018 
Figura 5. TSS (Training Stress Score)

150

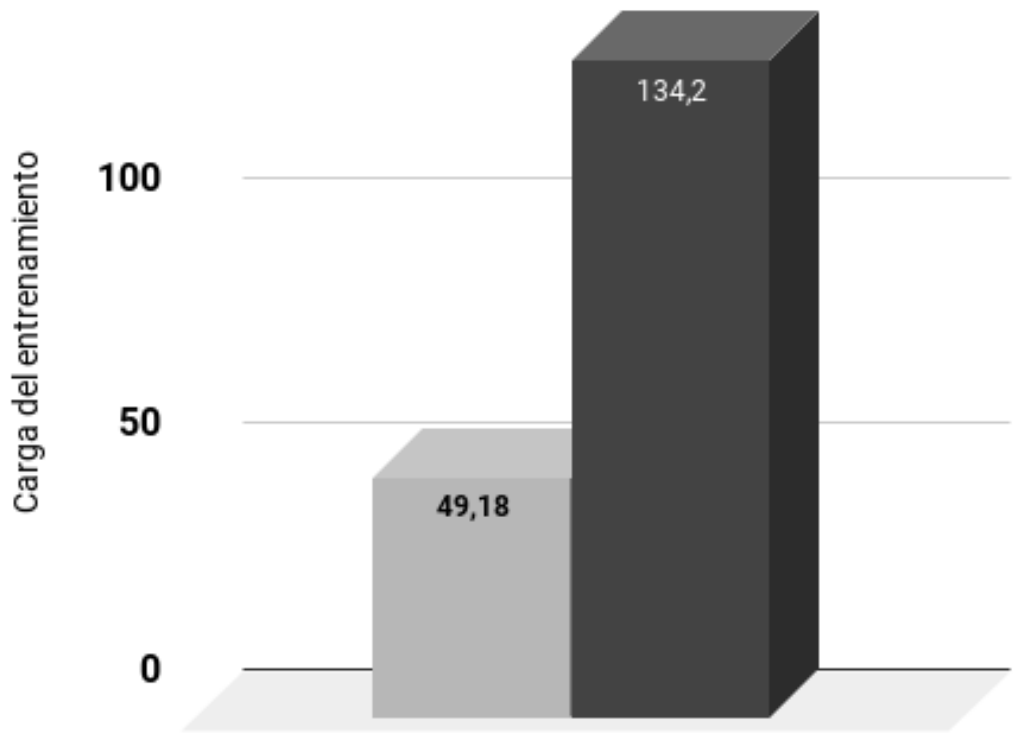

Febrero 2018 Enero 2019

Figura 6. Dolor pre-post ejercicio físico

10

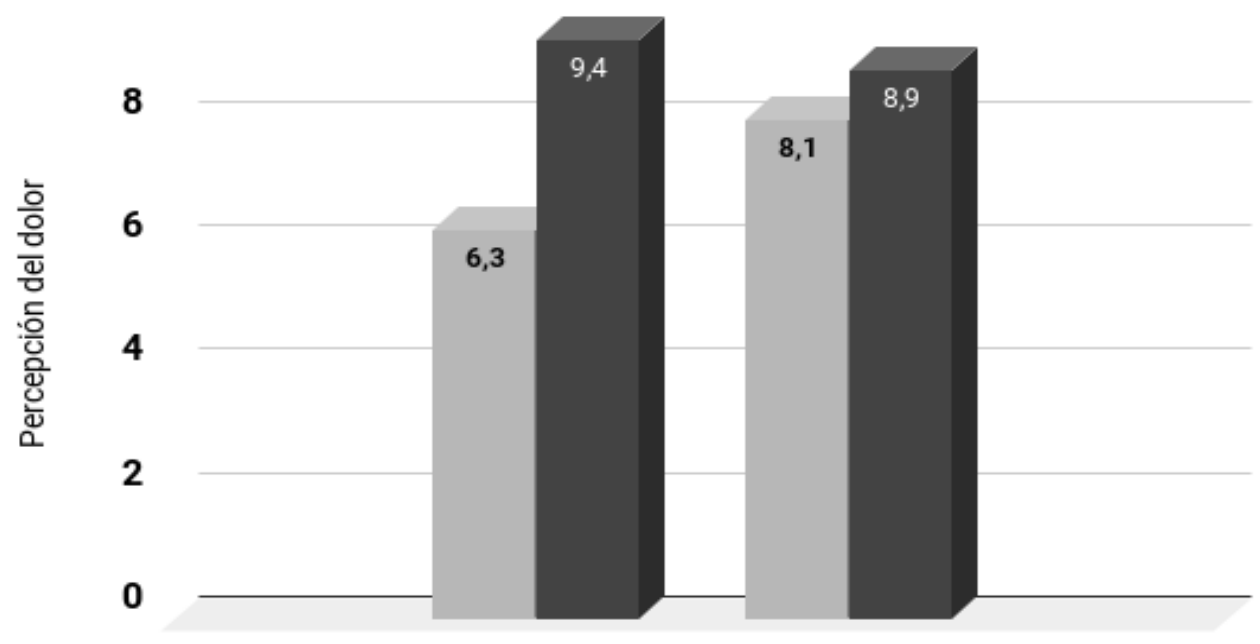

Febrero 2018

Enero 2019

107

informaciópsicoló Sica 
En lo que respecta al estado de experiencia óptima que experimenta durante los entrenamientos, las puntuaciones obtenidas son significativamente elevadas, con valores superiores a 10 tanto en antecedentes como en resultados del proceso, en una escala de 0 a 16.

En lo que respecta al dolor percibido antes y después de los entrenamientos, las puntuaciones del auto-registro correspondientes al mes de febrero de 2018 muestran cómo éste siempre es menor antes de realizar la actividad física que al acabar el entrenamiento. Es decir, el sujeto suele percibir más dolor tras el entrenamiento, salvo aquellos días en los que experimenta el mismo dolor insoportable tanto antes como después del entrenamiento.

Este posible efecto agudo de dolor tras los entrenamientos se invierte cuando comparamos parámetros relacionados con la salud durante periodos de tiempo más largos.

Por ejemplo, la medición del TSS o carga de trabajo asociada al ejercicio muestra que, en apenas un año, se triplica la fatiga asociada a las cargas de entrenamiento que el organismo es capaz de asimilar.

Tal y como podemos observar en la Figura 6, la media mensual del dolor percibido después de los entrenamientos en el periodo de un año parece indicar que, respecto del año anterior, éste ha disminuido.

\section{DISCUSIÓN Y CONCLUSIONES}

Pese a que la principal demanda del sujeto respecto de la intervención psicológica ha sido mantener o incrementar el rendimiento deportivo, el análisis de parámetros relacionados con la salud y con la evolución de los síntomas de la enfermedad parece mostrar cierta relación entre el ejercicio físico de alta intensidad y la mejora de los síntomas de la Esclerosis Múltiple, tal y como señalan recientes investigaciones (Rooney, Riemensschneider, Dalgas, Jorgensen, Michelsen, Brond y Hvid, 2019).

El incremento de la carga física (medida mediante el TSS) que puede asimilar el organismo del sujeto se triplica en apenas un año, en la línea de resultados basados en caminar (Kalron, Menascu, Frid, Aloni y Achiron, 2019), mientras que los dolores causados por esta enfermedad del sistema nervioso, pese a que se mantienen o incrementan de forma aguda tras el ejercicio, parecen reducirse a medio plazo. En la misma línea irían los datos de vatios promedio obtenidos durante los entrenamientos, que se incrementan con el transcurso del tiempo.

Con independencia de los resultados competitivos alcanzados por el sujeto fruto de la intervención, ajenos al objeto del presente estudio, cabe destacar la posible existencia de beneficios en la salud del deportista con EM, algunos de los cuáles identificables en programas de apenas unas semanas de implementación (Bahmani, Kesselring, Papadimitriou, Bansi, Püshe, Gerber, Shaygannejad, Holsboer-Trachsler y Brand, 2019).

Un dato aportado por el sujeto y su entorno familiar en esta línea podría ser la reducción en el número de crisis con ingreso hospitalario registradas entre los años 2017 y 2018 , pasando de 47 en el primero de ellos frente a tan sólo 2 en el segundo, respectivamente. Si bien no se puede establecer una relación causal, a nivel cualitativo si se han observado mejoras en el 
sujeto. En este sentido, el deportista informa de beneficios físicos y mejoras en relación con el número de ingresos, así como cambios positivos en los aspectos psicológicos. A modo de ejemplo podemos destacar verbalizaciones del tipo "cuando voy en la handbike me siento igual que los demás". Además, realiza atribuciones a una causas predecibles e identificable del dolor agudo frente al crónico, con manifestaciones como "cuando acabo el entrenamiento sé de dónde proviene el dolor que siento, al contrario que con los dolores neuropáticos", "cuando me esfuerzo mucho, las sensaciones desagradables que me producen las cargas de entrenamiento enmascaran los dolores cotidianos, y me olvido por un rato de ellos".

Por otra parte, las experiencias óptimas durante los entrenamientos podrían estar actuando como un elemento que favoreciera la adherencia al ejercicio físico, así como a que éste sea intensivo.

El estado de ánimo es un aspecto que oscila frecuentemente, especialmente sensible a situaciones interpersonales, y que se ve agravado por las dificultades económicas que genera en un sujeto incapacitado por la EM la necesidad de materiales y componentes (relacionados con la handbike, potenciómetro, ciclocomputador, desplazamientos, alojamientos, medio de transporte propio, etc.) que le permitan ser competitivo en las competiciones. Finalmente, el presente estudio, aunque tiene las limitaciones propias de una intervención en un caso único, muestra cómo la actividad física de alta intensidad es compatible con la esclerosis múltiple.

\section{Referencias}

Andrade, E., Arce, C., De Francisco, C., Torrado, J., y Garrido, J. (2013). Versión breve en español del cuestionario POMS para deportistas adultos y población general. Revista de Psicología del Deporte, 22(1), 95-102.

Bahmani, D.S., Kesselring, J., Papadimitriou, M., Bansi, J., Püshe, U., Gerber, M., Shaygannejad, V., Holsboer-Trachsler, E. y Brand, S. (2019). In Patients With Multiple Sclerosis, Both Objective and Subjective Sleep, Depression, Fatigue, and Paresthesia Improved After 3 Weeks of Regular Exercise. Frontiers in Psychiatry, 10, 1-9.

Balaguer, I., Castillo, I. y Duda, J. L. (2003). La Escala de Motivación Deportiva: una nueva medida de la motivación intrínseca, motivación extrínseca y no-motivación. Análisis psicométrico de la versión española. En Actas de resúmenes del II Congreso Internacional de Psicología Aplicada al Deporte (p.165). Madrid: Dykinson, S.L.

Balaguer, I., Castillo, I. y Duda, J. L. (2007). Propiedades psicométricas de la Escala de Motivación Deportiva en deportistas españoles. Revista Mexicana de Psicología, 24, 197-207.

Cantón, E. (2016). La especialidad profesional en Psicología del Deporte. Revista de Psicología Aplicada al Deporte y al Ejercicio Físico, 1, 1-12.

Dalgas U., Stenager E. y Ingemann-Hansen T. (2008). Multiple sclerosis and physical exercise: recommendations for the application of resistance-, endurance- and combined training. Multiple Sclerosis Journal, 14(1), 35-53

Dalgas, U., y Staneger, E. (2012). Exercise and disease progression in multiple sclerosis: can exercise slow down the progression of multiple sclerosis? Therapeutic Advances in Neurological Disorders, 5(2), 81-95. doi: $10.1177 / 1756285611430719$

McNair, D.M., Lorr, M. y Droppleman, L.F. (1971). Manual for the Profile of Mood States. California: Educational and Industrial Testing Services. McNair, D.M., Lorr, M. y Droppleman, L.F. (1992). Revised Manual for the Profile of Mood States. California: Educational and Industrial Testing Services. 
Ebers, G.C. (2008). Environmental factors and multiple sclerosis. The Lancet Neurology, 7, 268-277.

Esclerosis Múltiple España (2015). Informe del estudio Actividad Física y Deporte en Esclerosis múltiple. Madrid: Real Patronato de Discapacidad. Consultado el 28 de mayo de 2019. Disponible en: http://esclerosismultiple.com/ ftp publico/Informe $\% 20$ act $\% 20$ fisica $\% 20 y \% 20$ deporte $\% 20$ en $\% 20$ EM.pdf

FEDEMA (2015). Conocer la Esclerosis Múltiple. Sevilla: Federación de Asociaciones de Esclerosis Múltiple de Andalucía. Consultado el 20 de febrero de 2019. Disponible en: https://aedem.org/publicaciones/gu\%C3\%ADasy-manuales?download=137:conocer-la-esclerosis-m\%C3\%BAltiple-dirigido-a-personas-reci\%C3\%A9n-diagnosticadas-ed-fedema

Filipi M.L., Kucera D.L., Filipi E.O., Ridpath A.C. y Leuschen M.P. (2011). Improvement in strength following resistance training in MS patients despite varied disability levels. Neurorehabilitation, 28(4), 373-382.

García, T., Jiménez, R., Santos-Rosa, F.J., Reina, R., y Cervelló, E. (2008) Psychometric Properties of the Spanish Version of the Flow State Scale. The Spanish Journal of Psychology, 11(2), 660-669.

Kalron, A., Menascu, S., Frid, L., Aloni, R. y Achiron, A. (2019) Physical activity in mild multiple sclerosis: contribution of perceived fatigue, energy cost, and speed of walking. Disability and rehabilitation, 8, 1-7.

Moreno-Tenas, A. (2019). Intervención psicológica en ciclismo adaptado: Estudio de caso de un ciclista con esclerosis múltiple. Libro de actas del XVI Congreso de Psicología de la Actividad Física y Deporte, 529-532. Zaragoza: FEPD.

Motl R.W., Snook E.M., McAuley E. y Gliottoni R.C. (2006). Symptoms, self-efficacy, and physical activity among individuals with multiple sclerosis. Research in Nursing and Health 29(6), 597-606.

Motl, R.W. y McAuley, E. (2011). Association between change in physical activity and short-term disability progression in multiple sclerosis. Journal of Rehabilitation Medicine, 43, 305-310.

Negaresh, R., Motl, R.W., Zimmer, P., Mokhtarzade, M. y Baker, J.S. (2019). Effects of exercise training on multiple sclerosis biomarkers of central nervous system and disease status: a systematic review of intervention studies. European Journal of Neurology, 26(5), 711-721.

Núñez, J. L., Martín-Albo, J. y Navarro, J. G. (2007). Propiedades psicométricas de la versión española de la Escala de Motivación Deportiva. Revista de Psicología del Deporte, 16(2), 211-223.

Pelletier, L.G., Fortier, M.S., Vallerand, R.J., Tuson, K.M., Briere, N.M. y Blais, M.R. (1995). Toward a new measure of intrinsic motivation, extrinsic motivation, and amotivation in sports: The sport motivation scale. Journal of Sport and Exercise Psychology, 17, 35-53.

Rooney, S., Riemensschneider, M., Dalgas, U., Jorgensen, M.L., Michelsen, A.S., Brond J.C. y Hvid, L.G. (2019). Physical activity is associated with neuromuscular and physical function in patients with multiple sclerosis independent of disease severity. Disability and Rehabilitation, 41. DOI: 10.1080/09638288.2019.1634768

Ruíz, D. y Solar, L.A. (2006). Esclerosis múltiple. Revisión bibliográfica. Revista Cubana de Medicina General Integral, 22(2), 1-11.

Stuifbergen, A.K., Blozis, S.A., Harrison, T.C. y Becker, H.A. (2006). Exercise, functional limitations, and quality of life: a longitudinal study of persons with multiple sclerosis. Archives of Physical Medicine and Rehabilitation, 87, 935-943.

Tallner A., Waschbisch A., Wenny I., Schwab S., Hentschke C., Pfeifer K., y Mäurer, M. (2011). Multiple sclerosis relapses are not associated with exercise. Multiple Sclerosis Journal, 0(0), 1-4.

UCI (2019). Reglamento UCI del Deporte Ciclista. Aigle: Unión Ciclista Internacional. Consultado el 20 de febrero de 2019. Disponible en: https://s3-eu-west-1.amazonaws.com/static.yosoyciclista.com/documentos/smartweb/ menu/452/doc 5cac38d7c50366 15140931 16-Paraciclismo-act--20190201.pdf 